\title{
Benign Seminal Vesicle Neoplasm
}

National Cancer Institute

\section{Source}

National Cancer Institute. Benign Seminal Vesicle Neoplasm. NCI Thesaurus. Code C161643.

A non-metastasizing neoplasm that arises from the seminal vesicle. 